\title{
Introduction to Chapter 9
}

This chapter returns to a focus on a more personal writing style, documenting the experience of the authors over the past three to four decades with the different iterations and manifestations of action research, participatory action research and adaptive collaborative management. These authors are modest in their interpretations of their early experiences with these approaches, neglecting to mention that they were themselves part of the approaches' development. They describe the imprecise differentiation among AR, PAR and ACM as reflecting similarities in the three approaches.

This chapter also provides a good historical summary of the very influential approach taken in Nepal in the early years, an approach that influenced global ideas about participation and contributed to the development of global interest in community forestry and the value of forest user groups. Its emphasis on restoration/reforestation has good (and needed) lessons for current efforts in this arena.

The diversity of examples provided, from agriculture to water management, animal husbandry (nomadism) and forestry in a wide variety of locales and at various scales, is further testament to the flexibility and broad applicability of such approaches.

Fisher and Jackson also emphasize the importance of paying serious attention to understanding a context before diving into an ACM-like process. Specifically, they emphasize the value of ethnographic approaches for gaining such understanding.

We remember Fisher's suggestion, when we struggled with CIFOR's initial scepticism about ACM in the early 2000s, that we should focus on "plausible causal connections". Although we valued it as a "response" to critiques about lack of generalizability, Fisher suggested it to address our difficulties providing 'proof' of what we saw as demonstrable links between actions and impacts. 